Check for updates

Cite this: J. Mater. Chem. A, 2022, 10 2320

Received 23rd August 2021

Accepted 13th November 2021

DOI: $10.1039 / \mathrm{d} 1 \mathrm{ta} 07194 \mathrm{~g}$

rsc.li/materials-a

\section{Free standing dual phase cathode tapes - scalable fabrication and microstructure optimization of garnet-based ceramic cathodes $\dagger$}

\author{
Melanie Rosen, (D) ab Martin Finsterbusch, (D)*ac Olivier Guillon (D) ac \\ and Dina Fattakhova-Rohlfing *abc
}

\begin{abstract}
To make ceramic based all-solid-state batteries competitive for the battery market, a shift from the separator supported cell-design for lab cells to a scalable, cathode-supported one is necessary to improve the energy density. Using tape casting, we were able to demonstrate for the first time allceramic free-standing $\mathrm{LiCoO}_{2}(\mathrm{LCO}) / \mathrm{Li}_{6.45} \mathrm{Al}_{0.05} \mathrm{La}_{3} \mathrm{Zr}_{1.6} \mathrm{Ta}_{0.4} \mathrm{O}_{12}$ (LLZO) mixed cathodes with high capacities and active material utilization. Further morphology engineering by introduction of a sequential layer casting enabled us to tailor the microstructure of the mixed cathodes resulting in opposite concentration gradients for the active material and the electrolyte over the thickness of the cathode. With this optimized microstructure, we were able to increase the discharge capacity of the free-standing mixed cathodes to $2.8 \mathrm{~mA} \mathrm{~h} \mathrm{~cm}^{-2}$ utilizing $99 \%$ of the theoretical capacity. For the oxide garnet-based system, both the scalable fabrication method and the achieved electrochemical performance demonstrates industrial relevance for the first time. Additionally, the obtained free-standing cathodes have sufficient mechanical stability to allow the application of hybrid and ultra-thin separators to further increase the energy density on the full cell level.
\end{abstract}

\section{Introduction}

All-solid-state batteries based on garnet type $\mathrm{Li}_{7} \mathrm{La}_{3} \mathrm{Zr}_{2} \mathrm{O}_{12}$ electrolytes face many challenges in order to fully utilize their potential with respect to intrinsic safety and high energy density enabled by the use of metallic lithium anodes and high voltage cathode materials. Particularly, integration of solid electrolyte powders in the cell components and the scalable fabrication of cell designs providing maximum energy density on the cell level remain important issues to be addressed.

Practically all reported garnet-based cell architectures rely on the ceramic scaffolds as a mechanical backbone of the cell. In these so called separator-supported cells, sintered dense or porous garnet-based scaffolds are prepared in the first step, followed by an application of cathode layers and finally lithium metal anodes. The cathode half-cells often consist of single-phase thin cathode layers deposited on a dense planar garnet separator via physical processes such as pulsed laser deposition, ${ }^{\mathbf{1 , 2}}$ or via

${ }^{a}$ Institute of Energy and Climate Research - Materials Synthesis and Processing (IEK-1), Forschungszentrum Jülich GmbH, 52425 Jülich, Germany. E-mail: $m$. finsterbusch@fz-juelich.de

${ }^{b}$ Faculty of Engineering and Center for Nanointegration Duisburg-Essen, Universität Duisburg-Essen, Lotharstr. 1, 47057 Duisburg, Germany

${ }^{c}$ Helmholtz Institute Münster: Ionics in Energy Storage (IEK-12), Forschungszentrum Jülich GmbH, Corrensstr. 46, 48149 Münster, Germany

$\dagger$ Electronic supplementary information (ESI) available. See DOI: 10.1039/d1ta07194g wet-chemical coating. ${ }^{3}$ Even though such cells can achieve good cycling performance, the often low ionic and electronic conductivity of the cathode materials as well as processing restrictions limit the achievable layer thickness and areal capacity to the values below $0.1 \mathrm{~mA} \mathrm{~h} \mathrm{~cm}^{-2}$. Therefore, the fabrication of mixed cathodes, which incorporate a solid electrolyte to provide conducting pathways deep into the cathode layer, is necessary to successfully increase the cell capacity.

So far, two main routes have been reported to fabricate fully inorganic garnet-based mixed cathodes. The first route relies on the fabrication of a porous LLZO scaffold, which is subsequently filled with cathode active material by either sputtering ${ }^{5}$ or wet-chemical processes. ${ }^{4,6,7,8}$ Similar results can be obtained via the co-synthesis of nano-particulate LLZO and a cathode active material, typically $\mathrm{LiCoO}_{2}$ (LCO). While these approaches provide satisfactory electrochemical performance, the achievable loadings with cathode active material are low. The second approach therefore focuses on the co-processing and cosintering of LLZO and LCO. Many approaches include lithium borate (LBO) as a sintering agent either in a cathode slurry ${ }^{9-12}$ or a powder mixture, ${ }^{13}$ which is then coated onto a separator pellet. Thus, the sintering temperature can be significantly lowered to avoid the formation of secondary phases with unfavourable electrochemical properties. ${ }^{14}$ However, the low ionic conductivity of the LBO layer between the particles limits the effective ionic and electronic conductivity, as well as the capacity of the mixed cathodes. The introduction of advanced sintering 
techniques, such as field-assisted sintering (FAST), can also lower the sintering temperature while retaining the conductivity and capacity of the cells. ${ }^{15}$ Lastly, the careful control of the crystallinity of LCO as well as the sintering conditions during conventional sintering allowed for the successful manufacturing of an all-solid-state battery with a LLZO-LCO mixed cathodes with a capacity of over $1.5 \mathrm{~mA} \mathrm{~h} \mathrm{~cm}^{2}{ }^{16,17}$

In spite of the progress achieved in improving the performance of the fully inorganic cathode layers, the full-cell energy density in the separator-supported cells cannot be easily optimized as a certain separator thickness (in the range of several tenth of micrometres) is required to provide the mechanical stability of the cells. Much higher total cell capacities can be expected for the "cathode-supported" cell designs, in which a very thin (a few micrometre) separator layer would be deposited on a thick cathode layer with a high loading of cathode active material, as suggested for example by Rupp et al. ${ }^{18}$ The development of the cathode-supported cells heavily relies on the availability of free-standing mixed cathode layers with a high loading of cathode active material, which have not been reported yet. In view of the future large-scale cell production, scalable processing routes such as for example well established tape casting should be pursued to fabricate the cathode layers.

Besides the development of suitable processing protocols, optimization of the electrode morphology is essential to produce free-standing cathode layers with satisfactory performance. Thus, Finsterbusch and Danner et al. have shown that the capacity utilization is defined by a charge transport through a tortuous 3D structure of composite LCO/LLZO cathodes, which is strongly affected by the electrode thickness and the morphology of the cathode layers. ${ }^{16}$ They have suggested that a variation in the LCO to LLZO ratio can drastically improve performance, with a higher ionic conductivity (and thus solid electrolyte content) necessary at the separator side of the mixed cathode. It is therefore necessary to also control the microstructure of the mixed cathodes to achieve a full utilization of the cathode active material when the areal loading is further increased.

Based on the consideration above, this work aims at the fabrication of fully ceramic free-standing mixed cathodes. We show for the first time that the free-standing LCO/LLZO cathode layers with high capacities and active material utilization can be produced via a scalable tape casting procedure. Introduction of a sequential layer-casting enabled us to tailor the thickness and the microstructure of the mixed cathodes resulting in concentration gradients for the active material and the electrolyte over the cathode thickness. Investigation of the mixed cathode layers in the model cells clearly demonstrates the high impact of the microstructure on the capacity utilization, charging rate and the cycling stability of the mixed cathode layers, paving a way for the further optimization of the cathode performance.

\section{Experimental}

\section{Synthesis and powder conditioning}

$\mathrm{Li}_{6.45} \mathrm{Al}_{0.05} \mathrm{La}_{3} \mathrm{Zr}_{1.6} \mathrm{Ta}_{0.4} \mathrm{O}_{12}$ starting powder was prepared via solid-state synthesis. Stoichiometric amounts of $\mathrm{LiOH} \cdot \mathrm{H}_{2} \mathrm{O}$
(Merck, 98\%), $\mathrm{La}_{2} \mathrm{O}_{3}$ (Merck, 99\%, pre-dried at $900{ }^{\circ} \mathrm{C}$ for $10 \mathrm{~h}$ ), $\mathrm{ZrO}_{2}$ (Treibacher, 99.5\%), $\mathrm{Ta}_{2} \mathrm{O}_{5}$ (Inframat, 99.95\%) and $\mathrm{Al}_{2} \mathrm{O}_{3}$ (Inframat, 99.9\%) were used. $20 \mathrm{wt} \%$ excess of $\mathrm{LiOH}$ was added to compensate for lithium evaporation during hightemperature treatment. The mixture was thoroughly mixed (Retsch, 200M) and pressed into pellets before the first calcination step at $850{ }^{\circ} \mathrm{C}$ for $10 \mathrm{~h}$. The obtained pellets were crushed and ground by electrical mortar, pressed to pellets again and calcined a second time at $1000{ }^{\circ} \mathrm{C}$ for $10 \mathrm{~h}$. The pellets were again crushed and milled by mortar. The resulting LLZO powder shows cubic crystal phase with $\mathrm{Li}_{2} \mathrm{CO}_{3}$ surface impurities formed due to exposure to air, which decompose in the sintering process of the composite cathode.

To suitably reduce the particle size for further processing, the obtained powder was milled in ethanol with $\mathrm{ZrO}_{2}$ jar and $2 \mathrm{~mm}$ balls at $500 \mathrm{rpm}$ for $150 \mathrm{~min}$ using a planetary ball mill (Pulverisette 7 premium, Fritsch) and subsequently dried at $70{ }^{\circ} \mathrm{C}$ for $8 \mathrm{~h}$. To remove surface degradation (mainly $\mathrm{Li}_{2} \mathrm{CO}_{3}$ ) impurities from the LLZO surface, the dried powder was heated at $750{ }^{\circ} \mathrm{C}$ for $1 \mathrm{~h}$.

To reduce the particle size of the $\mathrm{LiCoO}_{2}$ (MTI), the received powder was milled in ethanol with $\mathrm{ZrO}_{2}$ jar and balls at $500 \mathrm{rpm}$ for 120 min using the same planetary ball mill and subsequently dried at $70{ }^{\circ} \mathrm{C}$ for $8 \mathrm{~h}$. All processing steps have been carried out in ambient air.

\section{Tape casting}

A solution of the dispersant (BYK 180, Altana), binder (PVB98, Sigma Aldrich) and additives (PEG400, Sigma-Aldrich and Solusolv, Solutia Inc.) in a mixture of ethanol (VWR, 99.6\%) and butanone (VWR, 99.6\%) was prepared. To prepare the slurry, the freshly heated LLZO powder and dried LCO powder were added to the solution and homogenized in a planetary mixer (Thinky) at $1500 \mathrm{rpm}$ for two minutes. Ratios of LLZO : LCO of $2: 1,1: 1$ and $1: 2$ by weight were used. The obtained slurry was cast onto a mylar foil. For the single cast layers, the gap height was fixed to $350 \mu \mathrm{m}$. For the sequentially cast samples, the gap height was adjusted to ensure a wet thickness of $200 \mu \mathrm{m}$ for all layers. After drying for $10 \mathrm{~h}$ at room temperature, the obtained green tapes were laminated under $125 \mathrm{MPa}$ at $80^{\circ} \mathrm{C}$ for $2 \mathrm{~min}$. After pressing, the green tapes were cut into $12 \mathrm{~mm}$ diameter discs, transferred into an $\mathrm{Al}_{2} \mathrm{O}_{3}$ crucible. The tapes were sintered on LLZO mother powder for $2 \mathrm{~h}$ at $1050{ }^{\circ} \mathrm{C}$ in air, with heating and cooling rates of $5 \mathrm{~K} \mathrm{~min}^{-1}$, to obtain free standing mixed cathodes. A detailed description of the preparation of LLZO separators can be found in our previous work. ${ }^{19}$

\section{Characterization}

The particle size distribution (ESI Fig. 1, $\uparrow$ top) of the starting powders was checked carefully via laser diffraction using a LA950 (Horiba Scientific) with a $650 \mathrm{~nm}$ and a $405 \mathrm{~nm}$ laser source. The data was analysed using a Mie model. The phase purity was measured using a Bruker D4 Endeavor equipped with a $1 \mathrm{D}$ detector LYNXEY using monochromatized $\mathrm{Cu} \mathrm{K} \alpha$ radiation. The microstructure was analysed using a scanning electron microscope (Hitachi, TM3000) on breaking edge as well as 
a wet-polished cross-section. The flatness of the samples was examined by white-light topography (Cyber Technology, CT300). To assemble the model cells, a gold current collector was sputtered onto the freshly sintered mixed cathodes $(\sim 30 \mathrm{~nm}$, Cressington 108cuto Coater). The free sides were coated with a solution of PEO (600 000, Sigma-Aldrich), LiTFSI (SigmaAldrich) and conductive carbon (Alfa Aeser) in acetonitrile. The ratio of $\mathrm{EO}: \mathrm{Li}^{+}$was fixed to 20 . The polymer solution was dried for $8 \mathrm{~h}$ at room temperature in argon atmosphere. The LLZO separators were heated to $750{ }^{\circ} \mathrm{C}$ for $2 \mathrm{~h}$ in argon to remove surface degradation layers. A thin gold interlayer was sputtered onto one side of the LLZO samples to ensure good contact with the metallic lithium. Freshly calandered metallic lithium was pressed on the coated side by hand, the sample then placed onto a $\mathrm{Ni}$ disc and the stack heated to $250{ }^{\circ} \mathrm{C}$ for $1 \mathrm{~min}$ to ensure good contact. The cathode and anode half-cells were stacked with the polymer layer in the middle to ensure fullsurface bonding, and the full cells were allowed to rest at $80^{\circ} \mathrm{C}$ for at least $24 \mathrm{~h}$ prior to measurement, to ensure full contact with the PEO and thermal equilibrium. All electrochemical measurements were carried out in a Swagelok cell at $80^{\circ} \mathrm{C}$. The impedance of the full cells was measured using a BioLogic VMP300 multipotentiostat. The frequency was varied from $7 \mathrm{MHz}$ to $1 \mathrm{~Hz}$ with an electrical field perturbation of $10 \mathrm{mV} \mathrm{mm} \mathrm{m}^{-1}$. Cycling tests of the full cells were carried out in a battery tester (Maccor) with either constant current-constant voltage mode (CC-CV) or constant current mode (CC) for charging and constant current (CC) for discharging. All cells were charged to $4.0 \mathrm{~V} v$ s. $\mathrm{Li} / \mathrm{Li}^{+}$at rates between $5 \mu \mathrm{A}$ and $25 \mu \mathrm{A}$. In case of CC$\mathrm{CV}$, the voltage was held until the current dropped from $25 \mu \mathrm{A}$ to $15 \mu \mathrm{A}$. All cells were discharged to $3.0 \mathrm{~V} v s$. $\mathrm{Li} / \mathrm{Li}^{+}$in CC mode at rates between $5 \mu \mathrm{A}$ and $25 \mu \mathrm{A}$, matching the corresponding charge rate.

\section{Results and discussion}

\section{Manufacturing of free-standing mixed cathode tapes}

The tape-casting process was designed to minimize the contact time between LLZO with ambient air and solvents, to minimize the $\mathrm{Li}^{+} / \mathrm{H}^{+}$-exchange, which is detrimental to the ionic conductivity. ${ }^{19}$ In total, three different free standing mixed cathodes based on LCO and LLZO were produced. First, the thickness of cathodes with a $1: 1$ weight ratio was controlled by laminating the dried green tapes. Two thicknesses were investigated, a thicker one featuring 4 layers, resulting in a mechanically very stable, all ceramic cathode with a thickness of $200 \mu \mathrm{m}$ and an areal loading of $32 \mathrm{mg} \mathrm{cm}^{-2}$ (Fig. 1a). Afterwards, the thickness was reduced to the point where the mechanical stability of the cathode still allowed for reliable cell assembly, resulting in just 1 layer with a final thickness of $70 \mu \mathrm{m}$ after sintering and an areal loading of $16 \mathrm{mg} \mathrm{cm}^{-2}$ (Fig. 1b). The diameter of all sintered cathodes was around $10.5 \mathrm{~mm}$.

Besides the thin and thick mixed cathodes with a homogeneous distribution of LCO and LLZO, a sequentially cast one with a variation in the active material to solid electrolyte ratio was produced (Fig. 1c). A first layer with a LLZO to LCO ratio of $2: 1$, a second layer on top of the first with a ratio of $1: 1$ and a third layer on top of these two with a ratio of LLZO to LCO $1: 2$ were cast in a sequential process. The casting gap and green tape thickness during the casting process were carefully controlled to ensure an even thickness of all three layers. The resulting mixed cathode has an average ratio of LLZO to LCO of $1: 1$. The overall thickness was adjusted to achieve the same loading as the thin, homogenous cathode produced before. The sintered gradient cathode features a gradient from 66 to $33 \%$ in the solid electrolyte content from the separator side to the current collector side. In contrast, the active material, which is also the electronic conductive phase, has the same gradient but in the opposite direction.

To validate the suitability of the produced mixed cathodes as battery components, their flatness, density and phase purity were examined. Microstructure analysis via scanning electron microscopy (SEM) (Fig. 1) shows a uniform distribution of both LLZO and LCO particles without large agglomerates for the thick, thin and gradient cathodes. All three mixed cathodes are well sintered, with mainly small pores. With an optimized sintering procedure, a relative density of over $90 \%$ was reliably achieved. Based on the energy dispersive X-ray analysis (EDX) measurements, the light particles and dark particles correspond to LLZO and the LCO, respectively (ESI Fig. 5a and $\mathrm{b}^{\dagger}$ ). No indication of secondary phases at the LLZO-LCO interface could be detected via SEM and EDX. After the temperature treatment, all cathodes remained suitably flat for the full cell assembly with only slight warpage on the edges (Fig. 2a). The relative density, determined by image analysis of the SEM pictures, is around $89 \%$. The thin and gradient cathodes display
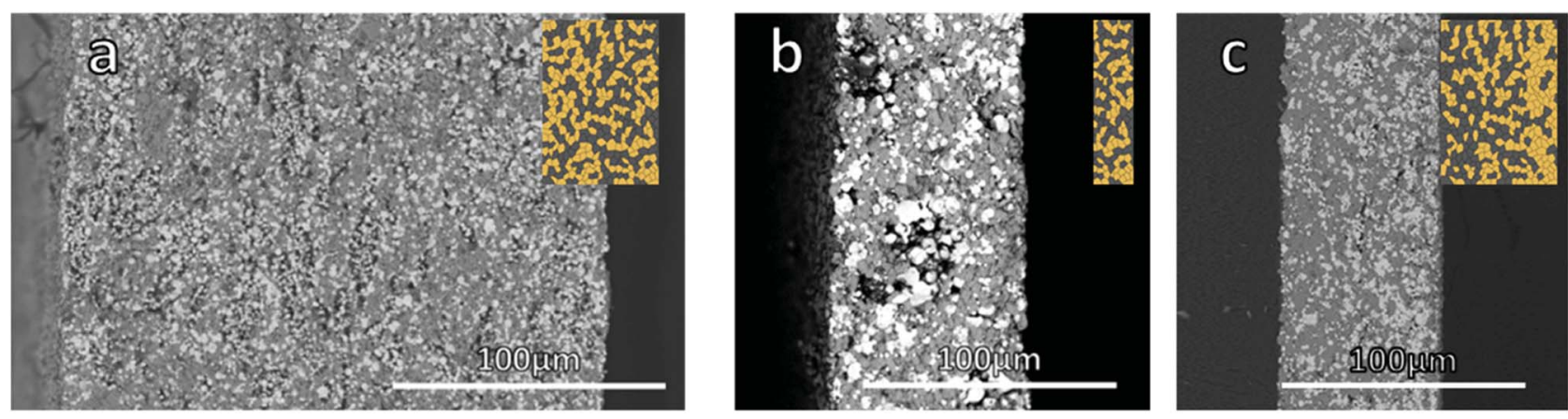

Fig. 1 Microstructure of the mixed cathodes from SEM images (a) thick cathode, (b) thin cathode, (c) gradient cathode. 

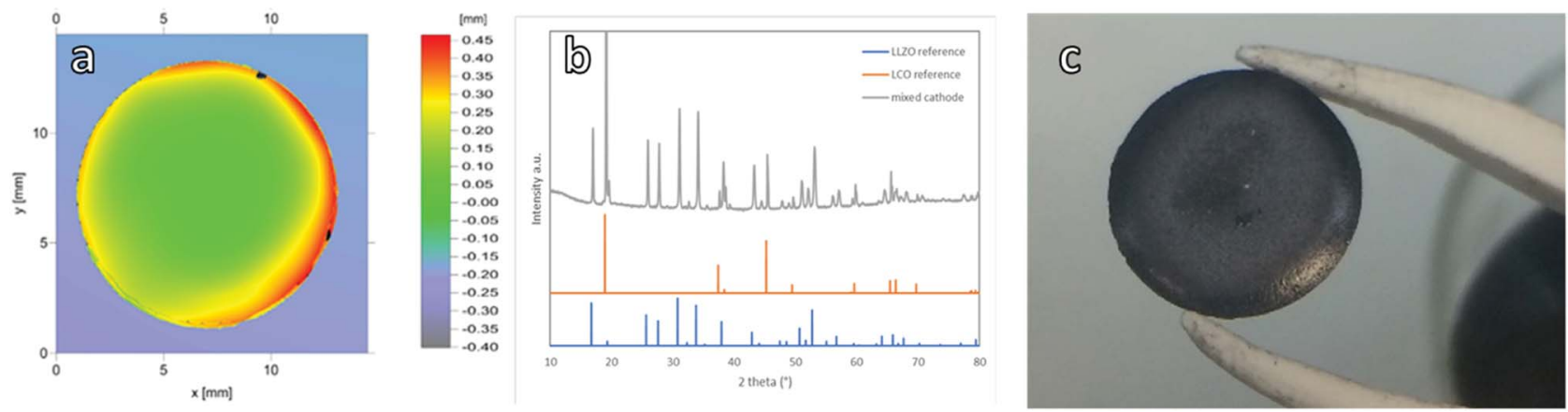

Fig. 2 Flatness and phase composition of a thick tape-cast LCO/LLZO layer after sintering at $1050{ }^{\circ} \mathrm{C}$ in air for $2 \mathrm{~h}$; (a) white-light topography; (b) XRD pattern (c) picture of free-standing mixed cathode.

a relative density of $92 \%$. The thick, thin and gradient cathodes display sufficient mechanical stability to withstand the stress during the cell assembly and operation (Fig. 2c). The weight of the mixed cathodes shows a variation of under $6 \%$ between cathodes, ensuring tight control over the active material loading of the cells. Since the secondary phase formation during thermal treatment was found to be one of the main reasons for poor performance in ceramic all-solid-state batteries, ${ }^{20}$ the prepared cathodes were examined via X-ray diffraction analysis (XRD). In the XRD patterns (Fig. 2b) only reflections assigned to LLZO and LCO were detected without indication of any secondary phases. Even though the detection limit of XRD is rather high (few wt\%) and thus the formation of minor amounts of side phases cannot be excluded based on the results of the XRD analysis, the obtained results demonstrate that the selected thermal treatment is generally suitable for the fabrication of mixed cathode without detectable processing-induced phase degradation.

\section{Model all-solid-state cells}

To investigate the electrochemical testing of the free-standing mixed cathodes they were incorporated into a model all-solidstate full cell. This model cell was specifically designed to enable the incorporation and comparison of different freestanding cathodes without an additional temperature treatment steps, thereby preserving the as-prepared state of the mixed cathode. We have to emphasize that the model cells are not designed to provide an optimized energy density on the cell level, but they enable a suitable platform for the evaluation and comparison of the performance of free-standing mixed cathodes.

A Li-metal anode in combination with a tape-cast LLZO separator of approx. $200 \mu \mathrm{m}$ thickness, as described in our previous work, ${ }^{19}$ provides protection against dendrite growth and a possible short-circuiting of the cell (Fig. 3c). With a suitable surface treatment and the incorporation of an alloying Auinterlayer, these separators allow for stable cycling up to $300 \mu \mathrm{A}$ $\mathrm{cm}^{-2}$, as reported in our previous work. ${ }^{19}$ To ensure good contact between the rigid, rough surfaces of the ceramic components, a thin interlayer of PEO:LiTFSI was introduced between the mixed cathode and the LLZO separator. The polymer electrolyte has an ionic conductivity of $9.810^{-4} \mathrm{~S} \mathrm{~cm}^{-2}$ at $80{ }^{\circ} \mathrm{C}$ (ESI Fig. $4 \dagger$ ), which is in a good agreement with the literature $^{21}$ and has been reported to show reasonably low contact resistances with both LLZO and LCO. ${ }^{22}$ A detailed examination of the cross-section via SEM (ESI Fig. $2 \dagger$ ) showed no infiltration of the polymer into the mixed cathode or LLZO separator due to their high relative densities.

To validate the suitability of the full-cell concept as a model cell, a detailed investigation of the impedance spectra of the as assembled and cycled cells was carried out on the thick cathodes. In both the as assembled and the cycled cells, three distinct semicircles can be identified (Fig. 3a). An analysis of the
ล

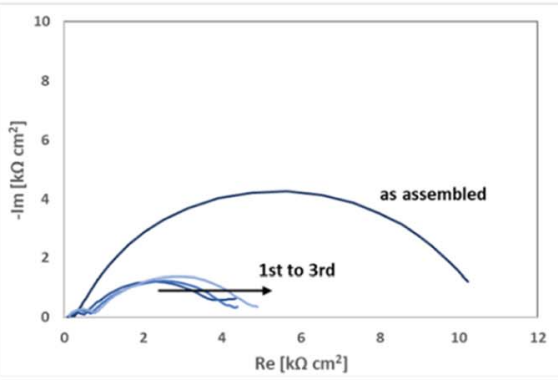

b

\begin{tabular}{lll} 
& As ass. & 1. cycle \\
\hline R1 $[\Omega]$ & 215 & 438 \\
C1 $[\mathrm{F}]$ & $4.710^{-9}$ & $1.510^{-9}$ \\
R2 $[\Omega]$ & 188 & 179 \\
C2 [F] & $0.1510^{-9}$ & $0.1210^{-9}$ \\
R3 $[\Omega]$ & 13700 & 4800 \\
C3 [F] & $0.6910^{-6}$ & $0.5910^{-6}$ \\
\hline
\end{tabular}

C

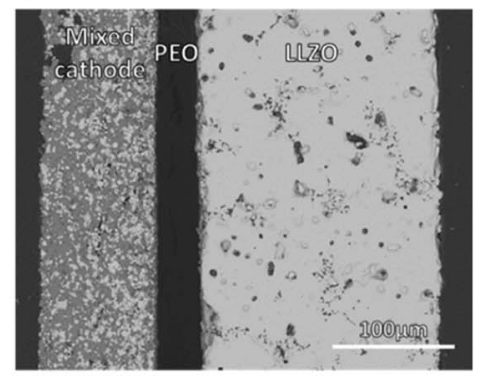

Fig. 3 (a): Impedance spectra at $80^{\circ} \mathrm{C}$ of the model cell including a thick cathode as assembled and after cycling; (b): fit of the impedance data of the as assembled cell and after the first charge-discharge cycle; (c): cross-section of the model cell. 
capacitance of the semicircles allows for an assignment to the phenomena in the cell. Both semicircles in the high frequency range have a capacitance in the $10^{-9} \mathrm{~F}$ order of magnitude and can therefore be identified as grain boundary resistances. During cycling, the R2 element does not change within the accuracy of the fit, therefore this resistance can safely be assumed to be related to the LLZO separator. This also matches the findings of our previous work on the similarly produced LLZO separators. ${ }^{19}$ In contrast, the resistance of the R1 element increases after the first cycle and continues to increase with growing number of cycles. As freely sintered mixed LCO/LLZO cathodes have been shown by us previously to degrade in another cell configuration, ${ }^{17}$ a rising resistance can be expected also for the free standing cathode tapes. The R1 element with can therefore be assigned to the mixed cathode grain boundaries. Finally, the R3 element shows a capacitance on the order of $10^{-6} \mathrm{~F}$ and can therefore be assigned to an interface resistance. As in our previous work, the Li-LLZO interface has been shown to have a resistance around $20 \Omega,{ }^{19}$ the R3 element has to be assigned to the interfaces of the PEO:LiTFSI electrolyte. After the first cycle the interface resistance has decreased by factor three. Whether this reduction stems from a better contact of the components due to the pressure and increased temperature or from the electrochemical reduction and formation of degradation products at the interface has to be further investigated.

\section{Electrochemical performance of free-standing mixed cathodes}

To benchmark the electrochemical performance of freestanding cathodes with different microstructure, the cells were charged and discharged at a constant rate. The voltage window was set within the stability window of the PEO:LiTFSI electrolyte and ranged from 3.0 to $4.0 \mathrm{~V} v s$. $\mathrm{Li} / \mathrm{Li}^{+}$.

For the $150 \mu \mathrm{m}$ thick mixed cathode without a concentration gradient, the current had to be limited to $5 \mu \mathrm{A} \mathrm{cm}{ }^{-2}$ to achieve successful charging and discharging due to its high internal resistance. This corresponds to a charge rate of $\mathrm{C} / 1000$ with respect to the theoretical capacity of $4.8 \mathrm{~mA} \mathrm{~h}$ (determined via the loading). The active material in the $150 \mu \mathrm{m}$ thick cathodes could not be fully utilized, as only $17.5 \%$ of the theoretical capacity (Fig. 4a) was achieved. These findings are in a good agreement with the simulations done by Finsterbusch and Danner et al., ${ }^{16}$ which predicted a sharp decrease in the performance for a mixed cathode of LLZO and LCO when their thickness exceeds $100 \mu \mathrm{m}$. When the cathode thickness increases, the lower effective ionic conductivity of the LLZO network causes large overpotentials and leads to insufficient (de-)lithiation of the LCO particles farthest away from the separator within the voltage limits.

For the thin mixed cathodes, the layer thickness of $70 \mu \mathrm{m}$ is lower than the theoretically calculated limiting electrode thickness. Due to the reduced internal resistance as compared to the thick cathode, the charge and discharge currents of the thin cathodes could be raised to $25 \mu \mathrm{A} \mathrm{cm}{ }^{-2}$, corresponding to $\mathrm{C} / 120$ charge rate with respect to its theoretical capacity of 3 $\mathrm{mA}$ h. Under these conditions, the cell shows an initial discharge capacity of $1.2 \mathrm{~mA} \mathrm{~h} \mathrm{~cm}^{-2}$ (Fig. $4 \mathrm{~b}$ ), or $74 \%$ of the theoretical discharge capacity (Fig. 5a), calculated from the active material loading.

The brittle nature of the free-standing mixed cathodes does not allow for the further reduction of the thickness and would conflict with the strife for high energy density cells. Thus, the Liion transport to the cathode active material furthest away from the separator has to be improved. ${ }^{16}$ For that, a higher effective ionic conductivity of the cathode layer has to be achieved. As the ionic conductivity of the cubic LLZO is limited to $1.84 \times$ $10^{-3} \mathrm{~S} \mathrm{~cm}^{-1}$ and no suitable ways to push it further were reported yet, ${ }^{23}$ alternative approaches should be investigated to improve the effective total electronic/ionic conductivity of the cathode layers such as adaptation of the microstructure. An approach similar to the multi-layer structures in solid oxide fuel cells, which accommodate the difference in oxygen partial pressure over the thickness of the cell, as well as the need for high ionic conductivities $^{29}$ has been introduced. The mixed cathodes produced via sequential tape casting feature a gradient in LLZO and LCO content. The microstructure was tailored to directly feature a higher effective ionic conductivity at the separator side of the mixed cathode by increasing the LLZO content in that layer. It additionally features a higher effective electronic conductivity on the current collector side of the mixed cathode by increasing the LCO content in that layer. Still, the overall content of cathode active material was kept at $50 \mathrm{wt} \%$. Thus, the problems addressed by Finsterbusch et al. can be accommodated without introducing new material into the system or lowering the cathode thickness and theoretical capacity.
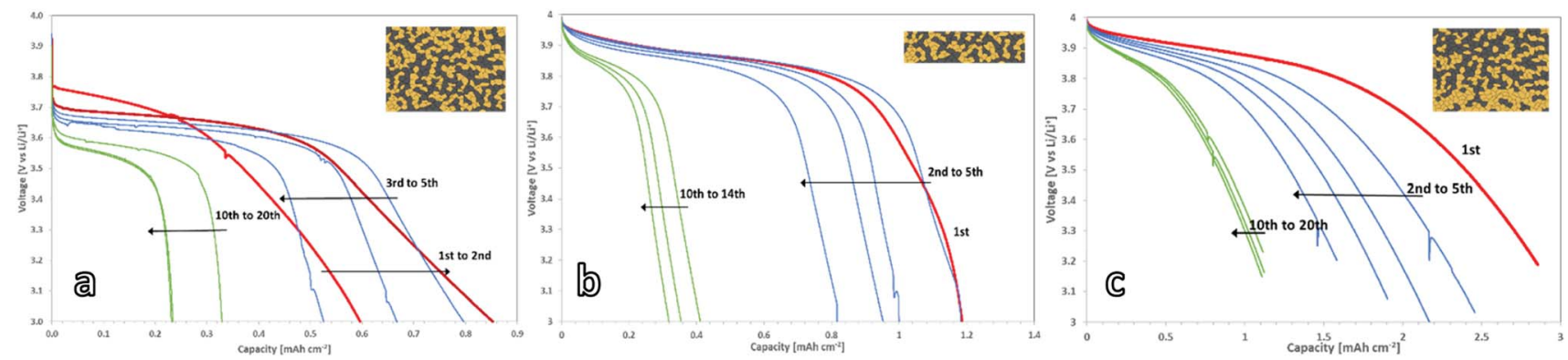

Fig. 4 Discharge curves of the cathodes with the similar loading of LCO but the different microstructure: (a) cell with a thick cathode, discharge rate $5 \mu \mathrm{A} \mathrm{cm}^{-2}$; (b) cell with a thin cathode, discharge rate $25 \mu \mathrm{A} \mathrm{cm}^{-2}$; (c) cell with a gradient cathode, discharge rate $25 \mu \mathrm{A} \mathrm{cm}^{-2}$. The measurements were taken at $80^{\circ} \mathrm{C}$. 

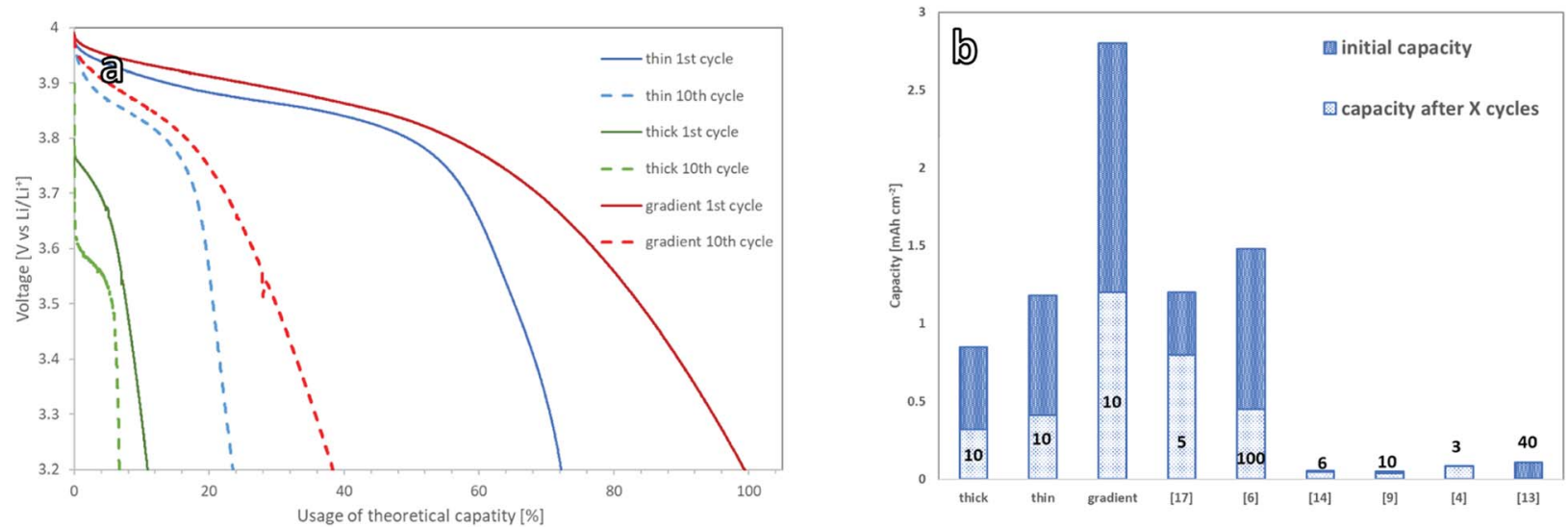

Fig. 5 (a) Discharge curves of thick (green), thin (blue) and gradient (red) mixed cathodes normalized for theoretical capacity; (b) literature overview of initial capacity and capacity after cycling for $X$ cycles.

A graded mixed cathode with approximately the same loading as the thin non-graded mixed cathode was produced to assess the impact of the gradient on the performance. It was also charged and discharged with $25 \mu \mathrm{A} \mathrm{cm}^{-2}$, corresponding to a $\mathrm{C} / 110$ charge rate in regard to the theoretical capacity of $2.8 \mathrm{~mA} \mathrm{~h} \mathrm{~cm}{ }^{-2}$, calculated from the active material loading. In the initial discharge cycle, $100 \%$ of the theoretical capacity is used (Fig. 4c). This marks the highest discharge capacity reported for a LLZO/LCO mixed cathode so far.

All three cathode concepts show degradation during the first cycles, leading to a coulombic efficiency of $90 \%$ during these cycles. After 10-20 cycles, a reduced but stable capacity with coulombic efficiencies over $98 \%$ is reached for all cathodes. The gradient cathodes with controlled microstructure are the most resistant to the degradation, retaining a capacity of $1.2 \mathrm{~mA} \mathrm{~h} \mathrm{~cm}^{-2}$ (Fig. 5a). As this is significantly higher than the retained capacity of the homogenous cathodes, the controlled microstructure can also tolerate the mechanical stress better.

The loss in full cell capacity is accompanied by an increase in cell resistance (Fig. 3a). This effect has previously been reported for all-ceramic cathodes and has been linked to the formation of micro-cracks, which form due to the volume change of LCO during charge and discharge. ${ }^{24}$ These cracks lead to a loss of electrical contact between the particles, rendering parts of the active material inaccessible. ${ }^{17,12}$ However, no mechanical degradation could be observed for the free-standing cathodes investigated in this work. The cathode tapes keep their mechanical integrity and no microstructure change or crack formation could be seen in the SEM images of the tapes after cycling (ESI Fig. $3 \dagger$ ). One of the reasons for the capacity fading could therefore be electrochemical degradation of the LCOLLZO interface as proposed by Park et al. ${ }^{25}$ which however has not been fully understood yet. The electrochemical oxidation of the PEO-LCO interface ${ }^{26}$ and the PEO itself can be also considered as the possible reasons of the observed degradation. Nevertheless, the capacity and cycling behaviour shown for our free-standing mixed cathodes in a model cell did outcompete high-capacity oxide solid-state cells, which rely on the separator as a mechanical support (Fig. 5b). ${ }^{15,17}$

The mechanical stability before and after electrochemical cycling renders the free-standing cathode tapes very attractive components for the cell development. The implementation of optimized anodes as described by Hitz et al. ${ }^{30}$ into the model cell will allow for cycling at higher current densities. Alternatively, the introduction of our free-standing cathode concept can provide the basis for thin-film separator coatings, yielding oxide based, high-energy density full cells. The reasons for the observed capacity fading should be however assessed further and mitigated to enable the all-ceramic cells with a high cycling stability. Similar concept of free-standing cathodes with a gradual microstructure can be applied to different material combinations with a higher energy density or higher electrochemical stability such as $\mathrm{NCM}^{27}$ or coated $\mathrm{LCO}{ }^{28}$ further increasing the electrode capacity and suppressing the degradation.

\section{Conclusions}

For the first time, free-standing oxide-ceramic mixed cathodes via tape-casting were demonstrated without the use of sintering additives. By incorporating the ceramic cathodes into a model cell, consisting of a reference anode side made from Li-metal, tape cast LLZO separator and a PEO interlayer, the electrochemical performance of the three produced cathodes was evaluated independently. By reducing the thickness and engineering the microstructure of the LCO-LLZO mixed cathodes via sequential tape casting, full cells with a capacity of $2.8 \mathrm{~mA} \mathrm{~h} \mathrm{~cm}^{-2}$ and close to $100 \%$ utilization of the active material in the first cycle were produced. This is the highest capacity of a fully ceramic mixed cathode so far. The free-standing mixed cathodes are able to provide sufficient mechanical stability for future solidstate batteries and can thus serve as the basis for the development of high-capacity cells with various thin separator concepts and anode designs, paving the way for cathode supported solidstate-cell concepts with competitive energy densities. 


\section{Author contributions}

Writing - original draft: M. R.; writing - review \& editing: M. F., O. G. and D. F.; investigation: M. R.; funding acquisition: M. F., D. F. and O. G.; project administration: M. F.; conceptualization: M. R., M. F. and D. F.

\section{Conflicts of interest}

There are no conflicts to declare.

\section{Acknowledgements}

This work was funded by the German Federal Ministry of Education and Research (BMBF) within of the Festbatt project (FKZ: 13XP0173A), EProFest project (FKZ: 13XP0346B) and the LISZUBA project (FKZ: 03XP0115B) and is gratefully acknowledged here. The authors would like to thank Philipp Hecker and Grit Häuschen for their help with the LLZO powder synthesis and heat treatment, and Dr Markus Mann for the SEM characterization.

\section{Notes and references}

1 S. Ohta, T. Kobayashi, J. Seki and T. Asaoka, J. Power Sources, 2012, 202, 332-335.

2 T. Kato, et al., J. Power Sources, 2014, 260, 292-298.

3 M. Kotobuki, H. Munakata, K. Kanamura, Y. Sato and T. Yoshida, J. Electrochem. Soc., 2010, 157, A1076.

4 K. J. Kim and J. L. M. Rupp, Energy Environ. Sci., 2020, 12, 4930-4945.

5 M. Kotobuki, K. Kanamura, Y. Sato and T. Yoshida, J. Power Sources, 2011, 196, 7750-7754.

6 M. Kotobuki, et al., J. Electrochem. Soc., 2010, 157, A493.

7 Y. Ren, T. Liu, Y. Shen, Y. Lin and C. W. Nan, Ionics (Kiel)., 2017, 23, 2521-2527.

8 M. Shoji, H. Munakata and K. Kanamura, Front. Energy Res., 2016, 4, 1-7.
9 S. Ohta, S. Komagata, J. Seki, T. Saeki, S. Morishita and

T. Asaoka, J. Power Sources, 2013, 238, 53-56.

10 F. Han, et al., Joule, 2018, 2, 497-508.

11 T. Liu, Y. Ren, Y. Shen, S. X. Zhao, Y. Lin and C. W. Nan, J. Power Sources, 2016, 324, 349-357.

12 T. Liu, et al., Electrochem. Commun., 2017, 79, 1-4.

13 S. Ohta, J. Seki, Y. Yagi, Y. Kihira, T. Tani and T. Asaoka, J. Power Sources, 2014, 265, 40-44.

14 L. J. Miara, W. D. Richards, Y. E. Wang and G. Ceder, Chem. Mater., 2015, 27, 4040-4047.

15 M. Ihrig, et al., J. Power Sources, 2021, 482, 228905.

16 M. Finsterbusch, T. Danner, C. Tsai, S. Uhlenbruck, A. Latz and O. Guillon, ACS Appl. Mater. Interfaces, 2018, 10, 22329-22339.

17 C. L. Tsai, et al., Sustainable Energy Fuels, 2019, 3, 280-291.

18 M. Balaish, J. C. Gonzalez-rosillo, K. J. Kim, Y. Zhu, Z. D. Hood and J. L. M. Rupp, Nat. Energy, 2021, 6, 227-239. 19 M. Rosen, et al., J. Mater. Chem. A, 2021, 9, 4831-4840.

20 L. Miara, et al., ACS Appl. Mater. Interfaces, 2016, 8, 2684226850.

21 R. Bouchet, S. Lascaud and M. Rosso, J. Electrochem. Soc., 2003, 150, A1385.

22 A. Gupta and J. Sakamoto, Electrochem. Soc. Interface, 2019, 28, 63.

23 L. Buannic, et al., Chem. Mater., 2017, 29, 1769-1778.

24 J. N. Reimers and J. R. Dahn, J. Electrochem. Soc., 1992, 139, 2091-2097.

25 K. Park, et al., Chem. Mater., 2016, 28, 8051-8059.

26 Z. Li, et al., Nano Energy, 2020, 72, 104655.

27 J. B. Goodenough and Y. Kim, Chem. Mater., 2010, 22, 587603.

28 Y. S. Jung, et al., Adv. Energy Mater., 2013, 3(2), 213-219.

29 E. D. Wachsman, et al., J. Electrochem. Soc., 1997, 144, 233236.

30 G. T. Hitz, et al., Mater. Today, 2019, 22, 50-57. 